\title{
Learning About Hydroelectric Power Plants Using a Model and Utilising a Game Scenario
}

\author{
http://dx.doi.org/10.3991/ijoe.v9i1.2403 \\ O. H. Graven and D.A.H. Samuelsen \\ Buskerud University College, Kongsberg, Norway
}

\begin{abstract}
The system described in this paper is focused on the challenge of engaging and motivating pupils in secondary schools and further education for STEM education in general, and demonstration of how energy conversion is done in hydroelectric power plants in particular. The method selected in order to engage and motivate the user is a games based approach. The implementation created to encourage the pupils to use the system and not only be passive viewers of a demonstration of the principles involved. The paper describes the design and implementation of the system. The system has been used by multiple groups of pupils over a period of two years.
\end{abstract}

Index Terms-Hydroelectric model; Games based Learning; promoting STEM education; inexpensive ADC.

\section{INTRODUCTION}

Over the last decade there is a necessary and on-going effort by many in attracting students to STEM (Science, Technology, Engineering and Mathematical) research. The system described in this paper is part of a project supported by local industry and part of a national focus in Norway to inform, educate and increase the interest in STEM education in general and engineering education in particular. The trend of relative interest in STEM is declining. Research from OECD[1] suggests it has been falling for prolonged periods across most of the developed world.

The project described is located within what is known in Norway as a Newton-room. The concept of Newtonrooms is rooted in the Norwegian Directorate of Education and Training. It has been developed over the course of several years by FIRST Scandinavia in cooperation with resource persons from schools and the industry. In the Newton-rooms, the students will become involved in running and exploring various aspects of STEM. There are Newton-rooms for energy, petroleum, chemistry, physics, mathematics, etc. An overview of all Newton-rooms in Norway can be seen in Figure 1.

The use of games for the presentation of technical content in a games context or the use of games to keep interest in a subject for longer periods of time is a method that, if used correctly can work. The project presented in this paper utilises such an approach.

The argument and motivation of the use of a game based approach can be summarised as follows: It will entice the users to spend more time interacting with the model, thereby gaining a better understanding of the basic principles involved. The game might also appeal to those users not primarily interested in technical issues, like planning for unknown future factors such as the fact that

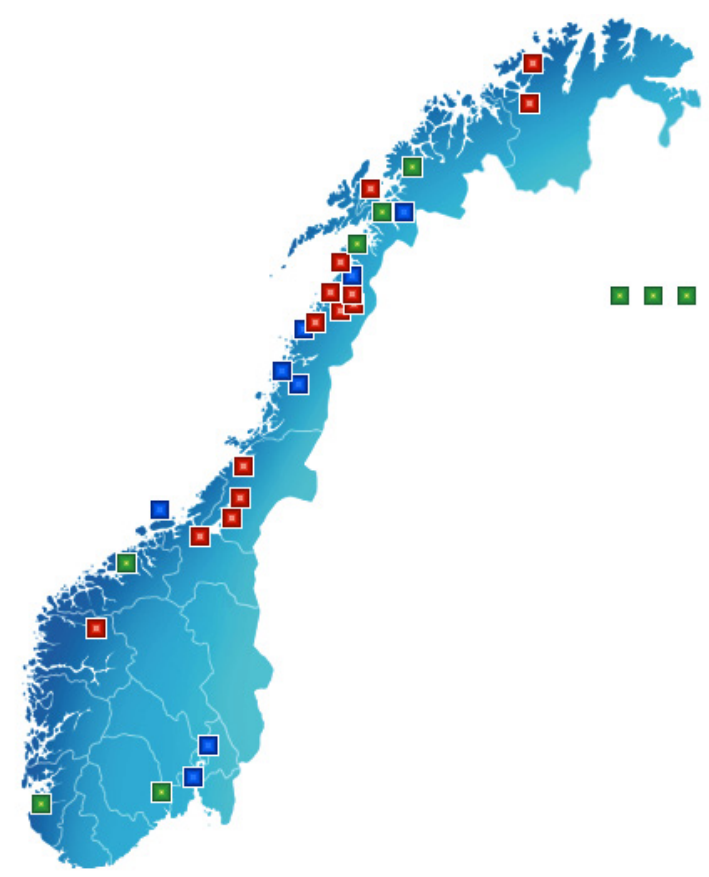

Figure 1. Overview of Newton-rooms in Norway

rainwater is a limited resource and varies from year to year, as is the power usage of a the consumers. In this way the game created will help by giving challenges as a multidisciplinary assignment: Technology inspired users and financially inspired user have to cooperate and understand the challenges for the other party.

\section{BACKGROUND}

\section{A. Game based learning}

As we know from school, young people are not always eager to do difficult things. The authors think that we as educators and tutors should learn from the gaming industry, which seems to have solved the challenge of getting new players to learn their games that are often long, complex and difficult, and even pay for the privilege. An interesting question is: How can we use this in education?

The decision to adopt a game-based approach to the development of e-learning follows on from a survey of current state-of-the art support for lifelong learning that was carried out by the authors[2], a key element of that investigation being the different techniques to improve learning and retention through engagement of the learner. In addition, Henderson[3] published a study showing that playing a recreational video game provided beneficial informal educative experiences, even when used only over 
PAPER

Learning About Hydroelectric Power Plants Using a Model and Utilising a Game Scenario

a short period of time. This study provides support for the cognitive worth of playing games, and it also contradicts the popular opinion by confirming that recreational video games are a significant cognitive artefact of youth culture.

Students learn more, and enjoy themselves more, when they are actively involved, rather than just passive listeners. Passive mode learning may seem the easy option for the students in the short run; everything is prepared and you just sit back and let it wash over you. Students who are not brought out of this passive state will usually learn little of the material thus presented, and will then tend to blame the tutor. In addition to make the students take an active role, education works best when it concentrates on thinking and understanding, rather than on rote memorization. Constructivism concentrates on learning how to think and understand.

The importance of setting a clear goal for students is also paramount. Once the goals are set, they help to keep students and content developers/lecturers focused on the items that were identified as important. One of the main difficulties students have is being able to separate the information that they really need to know from all the other less important information that is thrown at them. This is particularly important in an environment where the students are exploring. The content developers and lecturers must be aware of the dangers and make the environment as free of extraneous content as possible for the students. Scaffolding focused on giving the students a clear goal, a picture of what the expectations are, and where to focus their time and attention, will help their learning. Goals also give students something to strive for. This is important because it helps to motivate the student and it also provides a sense of accomplishment when goals are reached.

The system is created as a physical model of a simplified hydroelectric power plant, with a computerised control system for buying and selling electric power, and for general monitoring of the system. The control of water flow and thereby production of electricity is done manually by a valve the users have to turn. The reasoning behind a complete physical model and not just a computer simulation is that hands on experiences are important for the reality and the impressions that the users receive.

\section{B. Hydroelectric power "production"}

The main purpose of the system is to show that energy is converted rather that generated, due to the conservatism of energy in accordance with the first law of thermodynamics. The practical effect of this is that when more power is consumed by the "city", the loading of the generator is increased, resulting in more power being drawn from the turbine. In order to compensate for this, the water power pressure/flow has to be increased by the user controlling the valve. The result of this increased water pressure/flow in the game is that the reservoir is drained more quickly.

A secondary purpose of the system is to educate the users on the basic principles behind an actual hydroelectric power plant. Close to all electric power consumed in Norway is produced by hydroelectric power plants.

\section{DESIGN ASPECT}

The Newton room in Drammen is concerned with the handling of energy. Part one is the production or conver-

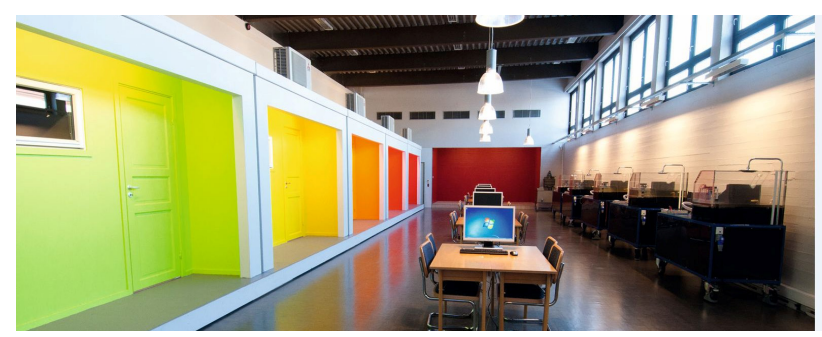

Figure 2. Inside the Newton-room in Drammen

sion of energy from potential/kinetic water energy toelectricity and the other part is concerned with the efficient use of the energy. The system described here is concerned with part one: the energy conversion. Figure 2 shows a picture of the setup with the energy converters, or hydroelectric power plant models to the right side and the model rooms with environmental controls for energy usage for lightning and heating/cooling on the left side.

\section{A. Requirements and limitation}

The two main requirements for the system are: 1 . It must be designed for all types of users from teenagers and upward, with a particular focus on groups of pupils in secondary and further education. 2. One of the major concerns is the security for the users. The system must be as safe as possible without a constant adult supervision. An adult will be present in the Newton-room at all times, but cannot be expected to close monitor all the groups simultaneously.

To accommodate various groups of users the system must be capable of varying the level of difficulty. The number of staff present at the Newton-room is small, one person in addition to the teacher that accompanies the pupils. The consequence of this is that the system must be quick and easy to setup, and maintenance must be easy, as there must be little down time.

To ease the use of the system a pack with information is distributed to the teachers as preparation before a visit to the Newton room. The teacher will prepare the pupils and themselves for the visit. This package also outlines suggestions for post-visit work and how the collected data can be reviewed. To enable the users to review and continue to work on the information gathered during the visit, a simple procedure must be available to export all collected data.

There is a desire to utilise techniques from games based learning in order to give the users an emotional experience linked to the task and thereby increase their motivation and engagement. An emotional attachment has been shown to increase the experience and provide emotional "hooks" for the users to better remember the information provided. [4]

\section{B. Content design}

The system is divided into three main parts: the physical water and production system, the power consumers, and the electronic monitoring system.

The computer control system controls the amount of rainfall available for power production, the power usage of the "city", and prices on the energy marked. The users have a set of controls available to them: A physical valve than can be turned and controls the flow and pressure of water on the turbine wheel mounted on the electric power generator, and the amount of power that is sold or bought 
PAPER

LeArning About Hydroelectric Power Plants Using a Model and Utilising a Game Scenario

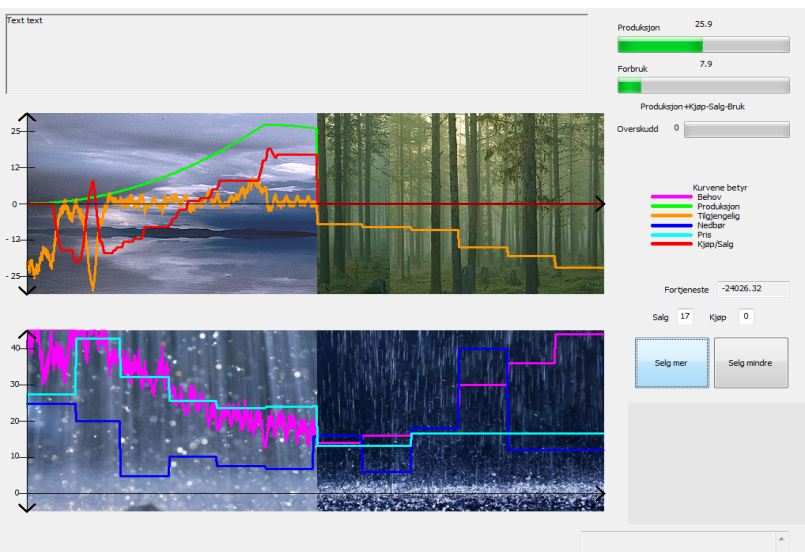

Figure 3. Screenshot half way into the game

off the energy market. The physical game element, the valve, is operated manually by a user, and their task is to match the production rate i.e. the flow/pressure of water, with the power consumption i.e. the loading of the hydro electric generator.

The computer part of the system has several tasks: Controlling the system, controlling the progression of the game, keep score, and display information to the users during the game.

The act of transforming the relatively simple task of controlling a valve into a game is done by introducing a few extra challenges for the users. The system displays an indication of future power demand based on an average obtained over multiple years, but will use an actual load that differs slightly from this, during the gameplay. In addition to future and real power demands, the system also controls the rainfall i.e. the amount of water available over time to produce electricity, and the prices of the energy marked.

Figure 3 shows the screenshot at the start of the game, Figure 4 shows how it may look about half way into the game. There are several indicators for the students to pay attention to:

- Top left there is a window showing three graphs: Production, amount bought/sold and surplus/lacking power (One aim is to keep this as close to 0 as possible).

- Bottom left there is a window showing three other graphs: Power requirement, rainfall, and price of power.

- Both windows have a background image, that is gradually changing showing the barrier between estimated future (right) and historic data (left)

- $\quad$ Top right there are 3 indicators showing: Production, Usage, and surplus/lack of power. All indicators also have a numeric value attached, to allow for accurate readings.

- Mid right are explanations for the users on what the different curves in the two main overview Windows show. This is in Norwegian.

- Bottom right there is an indicator of the running game score, amount currently bought or sold, and at the bottom two buttons to change the amount bought or sold. The buttons on the bottom are made large due to the use of a touch screen during the game. No mouse or keyboard is available.

- In the bottom right corner is the space where the dead fish appear, should the downstream river of the power plant run dry.

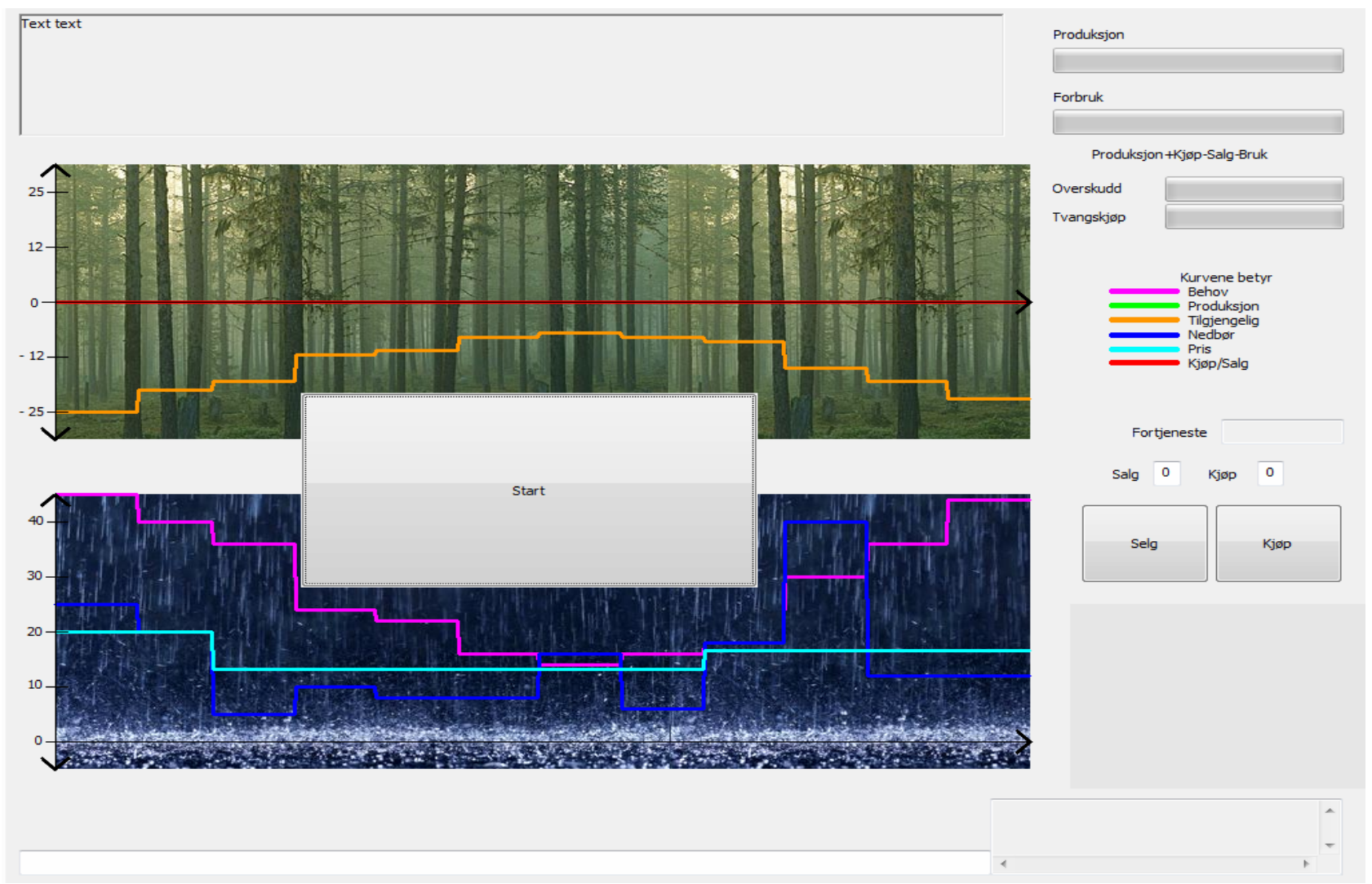

Figure 4. Screenshot of the application at the start 
The task for the users is to maximise profit over one year of game time, completed in less than one hour of real time. The planning involved in having the water resources available in times of high price and power use, is one of the challenges of the game. The introduction of buying and selling allows for a more advanced planning and potential higher profits, and will also introduce some challenges for the non-technically interested.

A few extra limitations are put into the game to make it model a real life production facility more correctly: There is a limited amount of storage space for water, and the rainfall and power usage does not follow the prognoses absolutely. In addition there are fishes downstream from the power plant that requires minimum amount of water flow, which allows for only very short periods of times with no production.

At the end of the game (Figure 5) there is an option to save all the results from the game into a excel style file so the pupils or teacher can take it with them and use theme for further discussion in the classroom when they come how to their own school.

\section{Implementation}

The implementation is completed using an industrial computer with touchscreen for user interaction, without mouse or keyboard is available for the users. Data acquisition and control of the hardware is done via a simple NIUSB6008 interface box.

The water reservoir is a placed on top of the model and is visible for the user at all times, but in order to increase the pressure for the turbine and to produce the rainfall i.e. bring water back to the reservoir, there is a pair of hidden pumps. The valve controlling the water pressure/flow is easily accessible on the model and located close to the turbine producing electricity. The water reservoir has an overflow "river" that bypasses the production facility. This can be seen in Figure 5.

Due to the safety requirements the system is designed using a nominal voltage of $12 \mathrm{~V}$ DC. Consumption of power is realised by an electronically controllable load resistor controlled by the computer. A map of the "city" and a set of LEDs showing parts of the "city" lighting up indicate actual power consumption for the user. Simultaneously the computer will show a set of graphs informing the users of predicted and actual power usage, prices, and rainfall.

\section{Challenge of measuring analogue signals}

The system utilises the simple NI-USB6008 interface box. This sampling solution requires analogue buffers for all the analogue input signals to be created in order to effectively reduce the internal resistance of the signal sources, in order to get correct measurements. All lowcost multichannel analogue acquisition solutions are built using a single analogue to digital converter (ADC) with the inputs connected via an analogue multiplexer. This is done so in order to reduce the cost of implementing expensive, high quality ADCs. Separate ADCs are only found in more expensive solutions when simultaneously sampling ADCs are required.

The practical consequence of this is that input buffers are required between each of the high impedance analogue signal sources and their respective analogue input to the ADC. The need for the buffers comes as a result of the

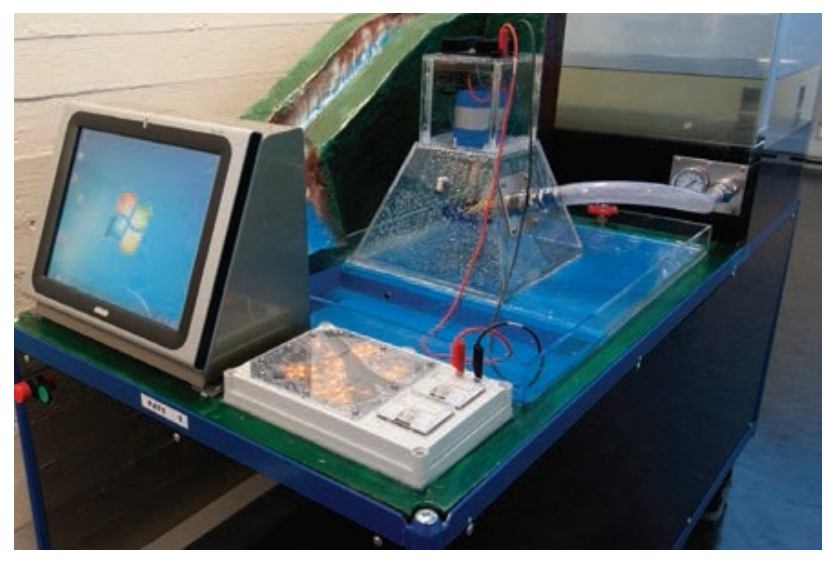

Figure 5. The hydroelectric system

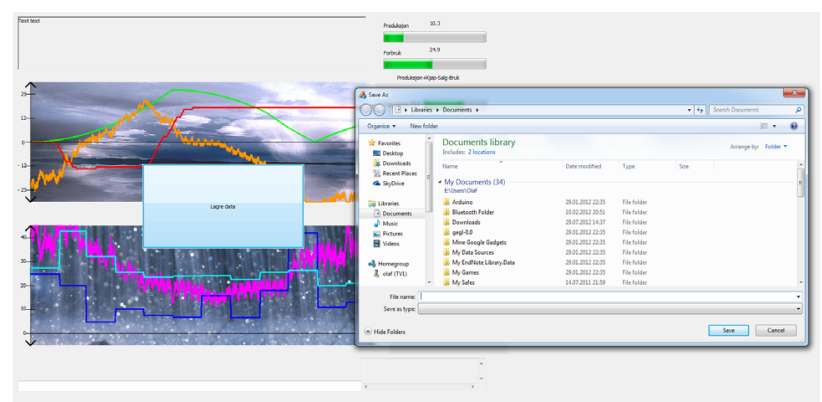

Figure 6. The end of the game

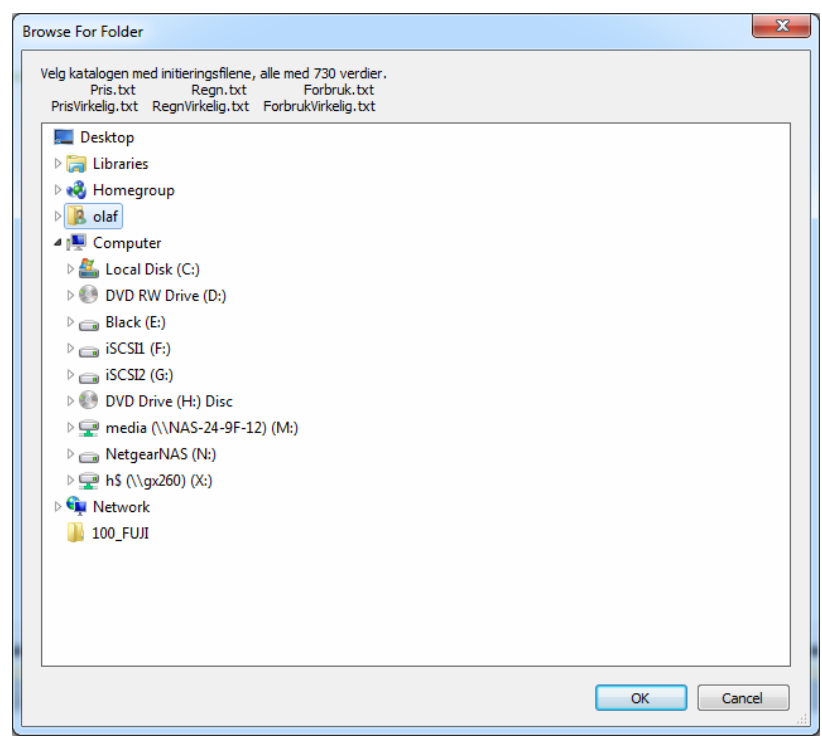

Figure 7. Selection of games setup files at the beginning of the game. (Typically done by the Newton-room teacher)

significant input capacitance of the sample-and-hold circuit placed after the analogue multiplexer in the ADC. Although the ADC has a high input resistance, giving an input bias current in the order of a few micro- or nano amperes, the dynamic characteristics of the analogue input causes the input impedance to low.

The result of this input capacitance is crosstalk between the input channels, meaning that if a signal source with low output resistance is connected to an input channel which is selected just before an input channel driven by a high output resistance source, the sensed signal of the latter will be highly influenced by the former signal. The 


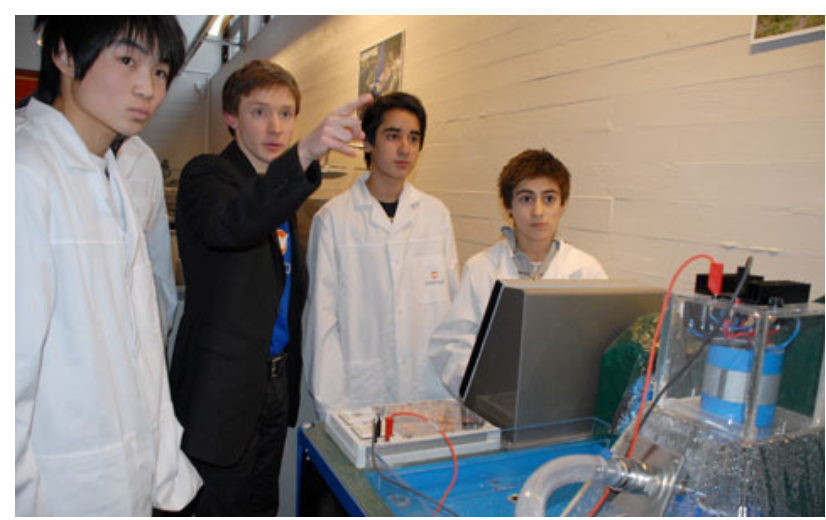

Figure 8. The system in use with 3 pupils and the Newton-room teacher

solution to this problem is to simply set up buffers with high input resistance and low output resistance, where the simplest version is the voltage follower. These buffers are physically located between the different signal sources and the NI-USB6008 interface box.

\section{USE OF THE SYSTEM}

The system has been used with some groups of pupils, Due to problem with the hardware producing the electrical power and the piping for the water, the system has been unreliable. In the occasions when the system has been in use, the feedback from the pupils was good. The Newtonroom teacher reports that the system is working and is motivating and engage the pupils to work with the system. They seem to bring with them an understanding on how the water is converted into electricity and the challenges of varying rainfall and power requirement.

There are multiple sets of power requirement, price, and rainfall created. This can easily be selected at the start up, see Figure 8. This gives the option for an easy, medium, or hard setup in the game. The feedback is that with the medium setup none have so far managed to end up with a positive score. There is even a group of pupils involved in the hardware production that has played many times in an effort to gain a positive score.

\section{CONCLUSIONS AND FURTHER WORK}

We have demonstrated a successful implementation of a games based system consisting of a computer controlled game and a physical model, educating users on energy conversion and hydroelectric power production. The fact that there is a group of pupils that when given the opportunity and access to the system return to play it again and again.

\section{ACKNOWLEDGMENT}

The authors would like to thank Buskerud fylkeskommune, Drammen kommune, ABB, Draka, EB, ECT, Elko, Otera Avotec, NITO Buskerud, Norsk Industri, InterProsjekt, Ing. Ivar Pettersen AS, Aker Solutions, Moelven, Høgskolen i Buskerud, and all secondary partners for the help in planning and implementing the Newton room in Drammen, Norway.

\section{REFERENCES}

[1] OECD, "Evolution of Student Interest in Science and Technology Studies Policy Report," OECD Global Science Forum Working Group2006.

[2] O. H. Graven and L. M. MacKinnon, "A survey of current state of the art support for lifelong learning," presented at the ITHET, Juan Dolio, Dominican Republic, 2005.

[3] L. Henderson, "Video Games: A Significant Cognitive Artifact of Contemporary Youth Culture," presented at the DiGRA, Vancouver, Canada, 2005.

[4] M. Sprenger, How To Teach So Students Remember: ASCD, 2005.

\section{AUTHORS}

O. H. Graven is with Buskerud University College, Kongsberg, Norway (Olaf.Hallan.Graven@hibu.no).

D. A. H. Samuelsen, is with Buskerud University College, Kongsberg, Norway (Dag.Samuelsen@hibu.no).

This article is an extended and modified version of a paper presented at the International Conference on Interactive Collaborative Learning (ICL2012), held 26 - 28 September 2012, in Villach, Austria.Received 30 Novemberr 2012. Published as resubmitted by the authors 24 January 2013. 